\title{
Exploration of bimetallic Au@Ag Core-Shell Nanocubes Dimers supports plasmonic Fano resonances
}

\section{Lamia Abu El Maati}

Princess Nora bint Abdulrahman University: Princess Nourah bint Abdulrahman University

Fatemah. H. Alkallas

Princess Nourah bint Abdulrahman University

Amira Ben Gouider Trabelsi

Princess Nourah bint Abdulrahman University

\section{Samira Elaissi}

Princess Nourah bint Abdulrahman University

\section{Tahani A. Alrebdi}

Princess Nourah bint Abdulrahman University

Mahmoud Ahmad ( $\nabla$ m.ahmad@mu.edu.sa)

Majmaah University https://orcid.org/0000-0001-6652-3419

\section{Research Article}

Keywords: dimers, Fano resonance, core/shell nanocubes, Plasmonic nanocavity

Posted Date: March 2nd, 2022

DOI: https://doi.org/10.21203/rs.3.rs-1375492/v1

License: (9) (i) This work is licensed under a Creative Commons Attribution 4.0 International License. Read Full License 


\title{
Exploration of bimetallic Au@Ag Core-Shell Nanocubes Dimers supports plasmonic Fano resonances
}

\author{
Lamia Abu El Maati (a), Fatemah. H. Alkallas ${ }^{(a)}$, Amira Ben Gouider \\ Trabelsi $^{(\text {a) }}$, Samira Elaissi ${ }^{(a)}$, Tahani A. Alrebdi ${ }^{(a)}$, Mahmoud Ahmad ${ }^{*}$ (b), (c)
}

(a) Department of Physics, College of Science, Princess Nourah Bint Abdulrahman University, P.O. Box 84428, Riyadh 11671, Saudi Arabia

(b) Physics Department, College of Science in Zulfi, Majmaah University, 11952, Saudi Arabia

(c) Physics Department, Faculty of Science, Al Azhar University, 71524 Assuit, Egypt. *corresponding author (Mahmoud Ahmad) E-mails m.ahmad@mu.edu.sa

\begin{abstract}
:
We report a theoretical simulation of optical spectra of Au@Ag core/shell nanocubes dimers. The influence of the core/shell size ratio and the dimers separation gap on these spectra have been studied using boundary element method (BEM). The scattering sections of various core/shell size ratio ( $=10 \%$ to $80 \%$ ) with different separation gap (2 to $16 \mathrm{~nm}$ ) have been numerically determined via Maxwell equations. We have examined the scattering sections along a wide wavelengths range, $250-1500 \mathrm{~nm}$, in visible and IR domain. The edge lengths of $60,80,100$ and $120 \mathrm{~nm}$ of the shell nanocubes have been considered. We revealed the formation of Fano resonance line shape through the investigation of the scattering section variation within the entire wavelengths. The Fano resonance line shape emerges with the variation of the gap between the dimer as well as of the core/shell size ratio. The trace of the Fano interference dip is slightly blue shifted and it is lost above gap separation equal $4 \mathrm{~nm}$. The core/shell dimer with varied core/shell size ratio manipulate and image easily the trace of the Fano resonance dip up to core/shell size ratio equal $50 \%$ for gap separation above $4 \mathrm{~nm}$. We demonstrate the important duality of the gap separation and core/shell ratio variation in designing Fano resonance interface. This has a potential effect on applications in nonlinear optics, metamaterial, tunable nanophotonic devices such as filters, wave-guiding, subwavelength optical imaging, chemical and biological sensing.
\end{abstract}

Keywords: dimers; Fano resonance; core/shell nanocubes; Plasmonic nanocavity 


\section{Introduction}

The plasmonic signature of gap cavity resonances modes has numerous applications in various disciplines, including nonlinear optics [1], metamaterials [2], tunable nanophotonic devices such as filters $[3,4]$ and plasmonic biosensors [5]. One of the promising gap cavity resonance mode is Fano resonance mode. Fano resonance mode appears when two resonances modes coupled together with noticeable difference of their linewidths [6]. Extensive attention is paid recently to explore Fano resonances, especially, those emerging due to a coupling between weak and large dipole moments [7]. Common research consider nanocavity made off noble metals to construct assemblies or units with various size, shape and orientation [8]. However, limited complex assembly shows a Fano resonance. Recently, Fano resonance were located in an individual silver nanocube isolated with a thin aluminum oxide from the supporting thin silver film. These structure were grown using a bottom-up method with controlled nanogap [9]. Many aspects of coupled plasmonic structures have been studied so far. Indeed, Fano resonance was found important for $\mathrm{Au}$ nanoplates placed on silicon layer. This effect is assigned to formation of $\mathrm{Au}$ nanosphere/Au nanoplate heterodimers. On other hands, Au nanosphere substitution with Au nanocube shows overwhelm of Fano resonance in the heterodimer. Such a resonance could emerge by modifying both of the nanoplate width and the nanosphere diameter [10]. Core/shell structure is one of the promising candidate in forming the gap cavity resonance mode. Plasmonic Fano resonances have been widely experimentally and theoretically studied. Particularly, those arise by core/shell nanorods made of gold core and silver shell $\mathrm{Au} @ \mathrm{Ag}$ nanorod [11], where they found Fano resonance located at elevated energies $(\approx 3.7 \mathrm{eV})$. Nanocavity can be established using nanocubes assembly. These structures enhance the field at their edges and importantly resonate due to the coupling with the placed metal substrates underneath [12]. Plasmonic oligomers i.e., dimers, trimers and tetramers play a major role as in emerging Fano resonance. These latest act as nanoclusters with hybridized eigenmodes powerfully related to the formations of the fundamental nanobars [13]. Fano resonance has been experimentally demonstrated via spatial modulation spectroscopy for silver and gold nanocube homodimers. Furthermore, numerical study investigating plasmon mode succeed to determine the principal mode implicated in the silver/ gold nanocubes homodimers. However, the inability of tracing the Fano resonance modes of higher order for a gap cavity below to $3 \mathrm{~nm}$ remains a hampering. Here, the Fano resonance mode either disappear or lost. On other hands, the phase shifts of the main Fano resonance modes of an homodimers with increasing of gap cavity from 2 to $16 \mathrm{~nm}$ are calculated [6]. In order to overcome this limit, Au@Ag core/shell nanocubes dimer are considered, where the impact of increase Au core size expected to play enhanced role of the main Fano resonance mode.

In this work, the influences of the gap separation on core/shell size ratio and the optical properties of Au@Ag nanocube dimers have investigated. The theoretical study of such properties has been determined using Maxwell's equations via boundary element method (BEM). This latest has a computational advantage in between of the two main types of numerical (Deferential, and integral) methods. Frequently presented deferential numerical 
methods are the finite deference time domain method (FDTD) [14], the finite elements method (FEM) [15] and the discontinuous Galerkin time-domain method [16]. Each deferential numerical method has its own limitation, For instance, FDTD discretizes in time domain with Yee's grid in space and the so-called leapfrog scheme [17], presenting a second-order accuracy. Due to its relaxed implementation and small computational difficulty, however, it also has revelation drawbacks in terms of numerical errors caused by staircase approximation of asymmetrical geometries and the constraint of CourantFriedrichs-Lewy condition (CFL) arising from the constancy problem in time domain [18]. Indeed, when modeling structures with curved boundaries, such as sphere and ellipse, FDTD employs controlled mesh to estimate the boundary. This results in a staircase-like estimation inducing errors to the original model. In contrast, the volume integral equations [19], the discrete dipole approximation (DDA) [20], the surface integral equations [21], and the boundary element method (BEM) [22] are types of popular integral numerical methods. The BEM method on electromagnetic field originated by charge distribution within a material and flow at its interface. It principally use solution of corresponding surface integrals by discretization with a number of representative points on the surfaces. This diminishes input as well as storage requirements for the final solution. Thus, a fast and operative determination of Maxwell's equations for truly arbitrary structures are ensured. The DDA divides the material into a grid of individual dipoles, for which the local field could be concerned as the summation of the external field and the field generated by all other dipoles. Both methods, BEM and DDA, deliver the resulting electromagnetic field in the frequency domain, whereby the surface parameterized with the BEM and the volume with the DDA.

BEM is used today in numerous areas of photonics, materials science, in both industrial and academic areas due to its high ability to predict and plan the optical properties of welldefined structures. The optical characteristics of metallic structures with numerous shapes and sizes have computed via BEM. This gains BEM method unlimited attention from diverse fields [23-25]. Amongst the fascinating nanostructures, nanocube allows high charges scatterings due to its sharp boundaries. Therefore, the exploration of near and far field properties of individual core/shell nanocube and core/shell nanocube dimer remains indispensable to the initial phases of synthesis and assemblies.

Herein, in this work, comprehensive analysis of $\mathrm{Au} @ \mathrm{Ag}$ core/shell nanocube dimers optical spectra dependency to both core/shell size ratio and separation gap between the dimer using BEM are performed. The scattering sections $\left(\mathrm{nm}^{2}\right)$ for different core/shell size ratio varying between $10 \%$ and $-80 \%$ for numerous gap separation ranges (i.e., from 2 to $16 \mathrm{~nm}$ ) have been established via Maxwell equations in the spectra domain [250 - 1500 $\mathrm{nm}]$. The edge length of 60, 80, 100 and $120 \mathrm{~nm}$ of the shell nanocube are considered. The scattering section variation within the entire wavelengths as a function of the gap between the dimer and of the core/shell size ratio are calculated. We admitted the description of boundary element method as developed in Ref [22] to our study.

\section{Numerical simulation}

Local and isotropic dielectric functions $\varepsilon_{j}(\omega)$ are considered to describe dielectric nanoparticles of single or many bodies $V_{j}$ with arbitrary shaped abrupt dielectric boundaries. The BEM ensures for structures of sharp edges owning dielectric sections of 
homogenous and isotropic dielectric function an appropriate Maxwell's equations resolution[26].

The electric and magnetic fields $\mathrm{E}$ and $\mathrm{H}$, respectively, could be determined via Helmholtz theorem to both scalar $\phi_{j}$ and vector $A_{j}$ potentials according to:

$E_{j}=i k A_{j}-\nabla \phi_{j}$

$\mathrm{H}_{j}=\frac{1}{\mu_{j}} \nabla \times \mathrm{A}_{j}$,

$\phi_{j}$ and $A_{j}$ are gathered through Lorenz gauge condition given by:

$\nabla \cdot \mathrm{A}_{j}=i k \varepsilon_{j} \mu_{j} \phi_{j}$

Maxwell equations could be expressed as:

$\nabla^{2} \phi_{j}+k^{2} \varepsilon_{j} \mu_{j} \phi_{j}=-4 \pi\left(\frac{\rho}{\varepsilon_{j}}+\sigma_{j}(s)\right)$

$\nabla^{2} \mathrm{~A}_{j}+k^{2} \varepsilon_{j} \mu_{j} \mathrm{~A}_{j}=-\frac{4 \pi}{\mathrm{c}}\left(\mu_{j} \mathrm{~J}+\mathrm{m}_{j}\right)$

$\sigma_{j}(s)=\frac{1}{4 \pi} D . \nabla \frac{1}{\varepsilon_{j}}, \mathrm{~m}_{j}=\frac{-1}{4 \pi}\left[i \omega \phi_{j} \nabla\left(\varepsilon_{j} \mu_{j}\right)+c \mathrm{H}_{j} \times \nabla \mu_{j}\right], k=\omega / c$ is the wavenumber in vacuum, $\varepsilon_{j}$ is the permittivity, $\rho$ is the charge density, $\mathrm{J}$ is the current density, $D_{j}=\varepsilon_{j} E_{j}$ represents the electric displacement, $B_{j}=\mu_{j} \mathrm{H}_{j}$ is the magnetic induction and $\mathrm{c}$ is the speed of light in vacuum. Both equations 4 and 5 are verified for laterally reliant dielectric permittivity. Regarding on sharp interface constrained two different media $\left(\varepsilon_{1}, \mu_{1}\right)$ and $\left(\varepsilon_{2}, \mu_{2}\right)$, the quantities $\sigma_{j}$ and $m_{j}$ are non-vanished only at the interface. Finding the suitable boundary conditions for electromagnetic field in the media split by abrupt boundaries the equivalent charge $\sigma_{j}$ and current $m_{j}$ can be calculated. These last equations could be resolved using Green function from the different sides of the boundary via:

$\left(\nabla^{2}+k_{j}^{2}\right) G_{j}\left(\mathrm{r}, \mathrm{r}^{\prime}\right)=-4 \pi \delta\left(\mathrm{r}, \mathrm{r}^{\prime}\right)$

where $k_{j}=k \sqrt{\varepsilon_{j} \mu_{j}}$ is the wavenumber in the medium, $\mathrm{r} \in V_{j}$ and $G_{j}\left(\mathrm{r}, \mathrm{r}^{\prime}\right)=\frac{e^{\left(i k_{j}\left|\mathrm{r}-\mathrm{r}^{\prime}\right|\right.}}{\left|\mathrm{r}-\mathrm{r}^{\prime}\right|}$

For an inhomogeneous dielectric situation, the solutions of Maxwell's equations may be written in ad-hoc form [22]:

$\left[\begin{array}{l}\phi_{j}(r) \\ A_{j}(r)\end{array}\right]=\int d r^{\prime} G_{j}\left(\left|r-r^{\prime}\right|\right)\left[\begin{array}{c}\frac{\rho\left(r^{\prime}\right)}{\varepsilon_{j}\left(r^{\prime}, \omega\right)} \\ \frac{J\left(r^{\prime}\right)}{c}\end{array}\right]+\oint d s G_{j}\left(\left|r-r^{\prime}\right|\right)\left[\begin{array}{c}\sigma_{j}(s) \\ h_{j}(s)\end{array}\right]$,

The scaler and vector potentials describing the external perturbation (e.g., dipole oscillation or plan wave) within a given medium $j$ are represented in the first term of RHS of Equation (7). At the boundaries, the discontinuity of Green function for the different existing 
permittivities are described by the second term of RHS. Herein, these functions are substituted with the effects of charges $\sigma_{j}(s)$ and currents distributions $h_{j}(s)$. Instead of integral notation in equation (7), compact matrix notation is used to connect directly BEM for numerical implementation, where each spatial dependency is divided into discrete number of the illustrative figures. With $\mathrm{j}=1,2$ where $\mathrm{j}=1$ denotes inside the particle and $\mathrm{j}=$ 2 denotes beyond the particle, the surface charges $\sigma_{j}$ and the currents $h_{j}$ are written as

$$
\begin{aligned}
& G_{1} \sigma_{1}-G_{2} \sigma_{2}=\phi_{2}^{e}-\phi_{1}^{e} \\
& G_{1} h_{1}-G_{2} h_{2}=A_{2}^{e}-A_{1}^{e}
\end{aligned}
$$

where $\phi_{j}^{e}$ and $A_{j}^{e}$ image the external disturbance affecting first terms of RHS in equation (7). The continuity of the potentials in equation (8) originates from the tangential component continuity of the electric field.. On other hands, Lorentz gauge condition and the dielectric displacement continuity results the following:

$$
\begin{aligned}
& H_{1} h_{1}-H_{2} h_{2}-i k \hat{n}\left(\varepsilon_{1} G_{1} \sigma_{1}-\varepsilon_{2} G_{2} \sigma_{2}\right)=\alpha, \\
& \alpha=(\hat{n} \cdot \nabla)\left(A_{2}^{e}-A_{1}^{e}\right)+i k \hat{n}\left(\varepsilon_{1} \phi_{1}^{e}-\varepsilon_{2} \phi_{2}^{e}\right) \\
& \varepsilon_{1} H_{1} \sigma_{1}-\varepsilon_{2} H_{2} \sigma_{2}-i k \hat{n}\left(\varepsilon_{1} G_{1} h_{1}-\varepsilon_{2} G_{2} h_{2}\right)=D^{e}, \\
& D^{e}=\hat{n} \cdot\left[\varepsilon_{1}\left(i k A_{1}^{e}-\nabla \phi_{1}^{e}\right)-\varepsilon_{2}\left(i k A_{2}^{e}-\nabla \phi_{2}^{e}\right)\right]
\end{aligned}
$$

Here $H_{1,2}=(\hat{n} . \nabla) G_{1,2} \pm 2 \pi$ represents the surface derivative associated with the Green function surface. Both equations (8) and (9) could be solved numerically using BEM to determine the surface charges and local currents. Here, the optical cross section per area element (da) could be established via the electromagnetic field through Poynting vector: as

$$
\begin{aligned}
& P_{s c a}=n_{b} \oint \operatorname{Re}\left(\hat{n}\left(\mathrm{E} \times \mathrm{B}^{*}\right)\right) d a \\
& P_{\text {ext }}=-\frac{1}{n_{b}} \oint \operatorname{Re}\left(\hat{n}\left(\mathrm{E} \times \mathrm{B}_{\mathrm{inc}}^{*}+\mathrm{E}_{\mathrm{inc}}^{*} \times \mathrm{B}\right)\right) d a \\
& P_{a b s}=P_{\text {ext }}-P_{s c a}
\end{aligned}
$$

where $n_{b}$ denotes dielectric background refractive index, and $\hat{n}$ denotes the outer surface vector at interface. The Poynting vector denoted by $(\mathrm{E} \times \mathrm{B}), \mathrm{E}_{\mathrm{inc}}$ and $\mathrm{B}_{\text {inc }}$ are the incident wave vector fields. The cross sections of Au@Ag nanocube dimer are computed using MNPBEM toolbox [26]. During computational procedures, the retardation effects have been considered. The nanocube unit of rounded edge is considered where default MNPBEM toolbox values of round-off parameter equal to 0.25 and grid size of 12 are used. We admit the formulated dielectric functions of gold and silver are of [27] in our calculations.

\section{Results and discussions}

To investigate the core/shell size ratio and the gap separation influence on of $\mathrm{Au} @ \mathrm{Ag}$ nanocube dimers optical properties, sequences of cross sections are calculated. The dependency of the core/shell size ratio and shell thickness of the considered dimers are 
shown in Table 1 [28]. As an illustration, Figure 1a show the cross sections for individual $\mathrm{Au} @ \mathrm{Ag}$ nanocubes of shell edge lengths of $60 \mathrm{~nm}, 80 \mathrm{~nm}, 100,120 \mathrm{~nm}$ at $10 \%$ core/shell size ratio. For this minimum core/shell size ratio, four main resonances modes D1, Q1, D2, and Q2 are labelled. The main dipolar resonance (D1) is the widest and weak in energy one reflecting the radiative characteristics, and the resonance D2 has antisymmetric charge distribution comparing to the median plane normal to the incident electric field, also it has dipolar characteristics. In contrast, the quadrupole resonances Q1 and Q2 arise from nonesymmetric charge distribution of the plane normal to the incident wave vector [6]. The resonances peaks of D1 and Q1 intercept and combine due to the inherent different symmetries. On the other hand, the dipolar resonances D1 and D2 interface together results in such none symmetric line-shape with scattering efficiency extinct in the short wavelength range. Such a behavior typically features Fano lineshape [29]. The optical spectra of Au@Ag core/shell nanocubes dimers of $60 \mathrm{~nm}, 80 \mathrm{~nm}, 100$ and $120 \mathrm{~nm}$ variation comparing to of the core/shell size ratio and the gap separating dimers are calculated. In order to discuss the differences between individual $\mathrm{Au} @ \mathrm{Ag}$ nanocube and $\mathrm{Au} @ \mathrm{Ag}$ nanocube dimer of various gap separation, the cross sections of core/shell nanocube dimers are calculated at different gap separations Fig 2a. For the large gap separation d=20 nm Fig 2a, the cross sections of $\mathrm{Au} @ \mathrm{Ag}$ core/shell nanocube dimer are not much different from the individual Au@ Ag core/shell nanocube at the minimum core/shell size ratio except for noticeable red shift of the whole spectrum and rise of the main resonance mode linewidth. The main four resonances modes D1, Q1, D2, and Q2 of the individual Au@ Ag nanocube are compared to the related four main hybridized resonances modes, 1 (D1-D1), 2 (Q1Q1), 3 (D2-D2), and 4 (Q2-Q2) of the Au@ Ag nanocube dimer, the hybridization effects and the overlaps of individual LSPR have weak effect for such large gap separation. Fig $1 \mathrm{~b}$ illustrates the field distribution enrichment particularly of that close to $\mathrm{Au} @ \mathrm{Ag}$ nanocube, while the norm of electric field distribution (A E F) and Fig 2b show the corresponding of $\mathrm{Au} @ \mathrm{Ag}$ nanocubes dimers. The field enhancement distributions of $\mathrm{Au} @ \mathrm{Ag}$ nanocube dimer reflects the signature of the individual Au@Ag nanocube D1, Q1, and D2 modes without any complex alteration except for noticeable higher norm of electric field. For smaller gap separation $(\mathrm{d}=2 \mathrm{~nm})$, stronger modes hybridizations are identified. Fig 3a show clear red shift of the whole spectrum, mainly for the main resonance mode 1 for all dimers under consideration and decreasing of the linewidths of the main resonance mode is noticed, however the remained three modes 2,3 , and 4 are slightly red shifted. The remarkable aspects are the two resonances modes G1 and G2 where, G1 appear as a peak in the extinction cross sections spectrum, while $\mathrm{G} 2$ appear as a dip which explains the transparency window observed in [30]. The coupling of dipolar modes with the G2 resonance dip is accountable for the creation of Fano interference shape, where the induced transparency as well as Fano interference is formed when two resonances modes coupled together with noticeable difference of their linewidths. Both the two resonances modes G1 and G2 are red shifted when the size of the dimer increased, also their intensities and the spectral width are slightly increased. Fig $3 b$ show the related field enhancement and the norm of electric field (A E F) at small gap separation (d=2 nm) of Au@ Ag nanocube dimer. In comparison of large gap separation, clear charge distributions change and noticeable 
high norm of electric fields are shown. In order to trace the Fano interface dip of $\mathrm{Au} @ \mathrm{Ag}$ nanocube dimer, the scattering cross sections of $\mathrm{Ag}$ shell equal $100 \mathrm{~nm}$ at minimum core/shell ratio of gap separation vary from 2 to $16 \mathrm{~nm}$ are presented. Fig 4 show the tracing of G2 interface dip with the variation of the gap separation. The trace of the G2 interface dip is lost and slightly blue shifted above gap separation equal $4 \mathrm{~nm}$. In order to overcome this limit, the core shell size ratio is increased from 10\% to 80\%. Fig 5-7 present the calculated scattering cross sections of Au@Ag nanocube dimer of different shells sizes of $60 \mathrm{~nm}, 80 \mathrm{~nm}, 100 \mathrm{~nm}, 120 \mathrm{~nm}$ at different core/shell size ratio between $10 \%$ to $80 \%$ of gap separation equal 2, 4, $6 \mathrm{~nm}$ respectively. Tacking the advantages of such heterodimers with varied core/shell size ratio, it can easy trace and manipulate the Fano resonance dip up to core/shell size ratio equal 50\% for any gap separation. In addition, a new remarkable resonance peak labeled as G0 is formed with the variation of core/shell size ratio. The G0 resonance peak is originated from dipolar coupling of $\mathrm{Au} / \mathrm{Au}$ core/core inner dimer alone and it is blue shifted for higher of core/shell size ratio and it is red shifted when the outer dimer separation is increased. The effect of G0 resonance peak on G2 Fano resonance dip, Fig S1-S8 (supporting information) show scattering cross sections of Au@ Ag nanocube dimer of different shells size of $60,80,100$, and $120 \mathrm{~nm}$ variation with the core/shell size ratio. As shown in Fig S1-S8, The impact of increasing core/shell size ratio on the stability overall spectrum and tracing of Fano resonance dip up to 50\% core/shell size ratio of all dimers under consideration. For elevated core/shell size ratio greater than $50 \%$ and the main resonance peak (1) is at maximum, the tracing of Fano resonance dip is lost again. Details on scattering cross section of Au@Ag nanocube dimer of different shells size of 60, 80, 100, and $120 \mathrm{~nm}$ as a function of dimer gap are shown in Fig S9-S16.

\begin{tabular}{|l|l|l|l|l|l|l|l|l|}
\hline A (nm) & B (nm) & C (nm) & D (nm) & E (\%) & F (nm) & G (nm) & H (nm) & I (nm) \\
\hline 12 & 10 & 8 & 6 & $10 \%$ & 54 & 45 & 36 & 27 \\
\hline 24 & 20 & 16 & 12 & $20 \%$ & 48 & 40 & 32 & 24 \\
\hline 36 & 30 & 24 & 18 & $30 \%$ & 42 & 35 & 28 & 21 \\
\hline 48 & 40 & 32 & 24 & $40 \%$ & 36 & 30 & 24 & 18 \\
\hline 60 & 50 & 40 & 30 & $50 \%$ & 30 & 25 & 20 & 15 \\
\hline 72 & 60 & 48 & 36 & $60 \%$ & 24 & 20 & 16 & 12 \\
\hline 84 & 70 & 56 & 42 & $70 \%$ & 18 & 15 & 12 & 9 \\
\hline 96 & 80 & 64 & 48 & $80 \%$ & 12 & 10 & 8 & 6 \\
\hline
\end{tabular}

Table 1 illustrated the impact of the core/shell size ratio and shell thickness on the investigated dimers. The columns (A-D) correspond to the edge length (nm) of Ag shell of edge lengths of 120,100, 80 and $60 \mathrm{~nm}$ respectively. Column (E) indicates the utilized core/shell size ratio, and columns (F-I) is assigned to the obtained shell thicknesses in (nm) of $120,100,80$, and $60 \mathrm{~nm}$ correspondingly. The shell thickness $(\mathrm{t})$ is obtained via $\mathbf{t}=(\boldsymbol{a}-$ b)/2 where a, b designate to the edge length $(\mathrm{nm})$ of the shell and core.

\section{Conclusion}

In summary, plasmonic Au@Ag core/shell nanocubes dimers with varied separation gap and with different core/shell size ratio has been investigated. As the gap between the dimer is varied, Fano resonance interference is formed. The trace of the Fano interference dip is 
slightly blue shifted, and it is lost above gap separation equals $4 \mathrm{~nm}$. Tacking the advantages of such core/shell dimers with varied core/shell size ratio, we found that, it can easy trace and manipulate the Fano resonance dip up to core/shell size ratio equal $50 \%$ for gap separation up to $14 \mathrm{~nm}$. Our finds are beneficial to clarify Plasmon resonances of bimetallic structure that may address Fano resonance interface for several potential applications in nonlinear optics, metamaterial, tunable nanophotonic devices.

Figures captions
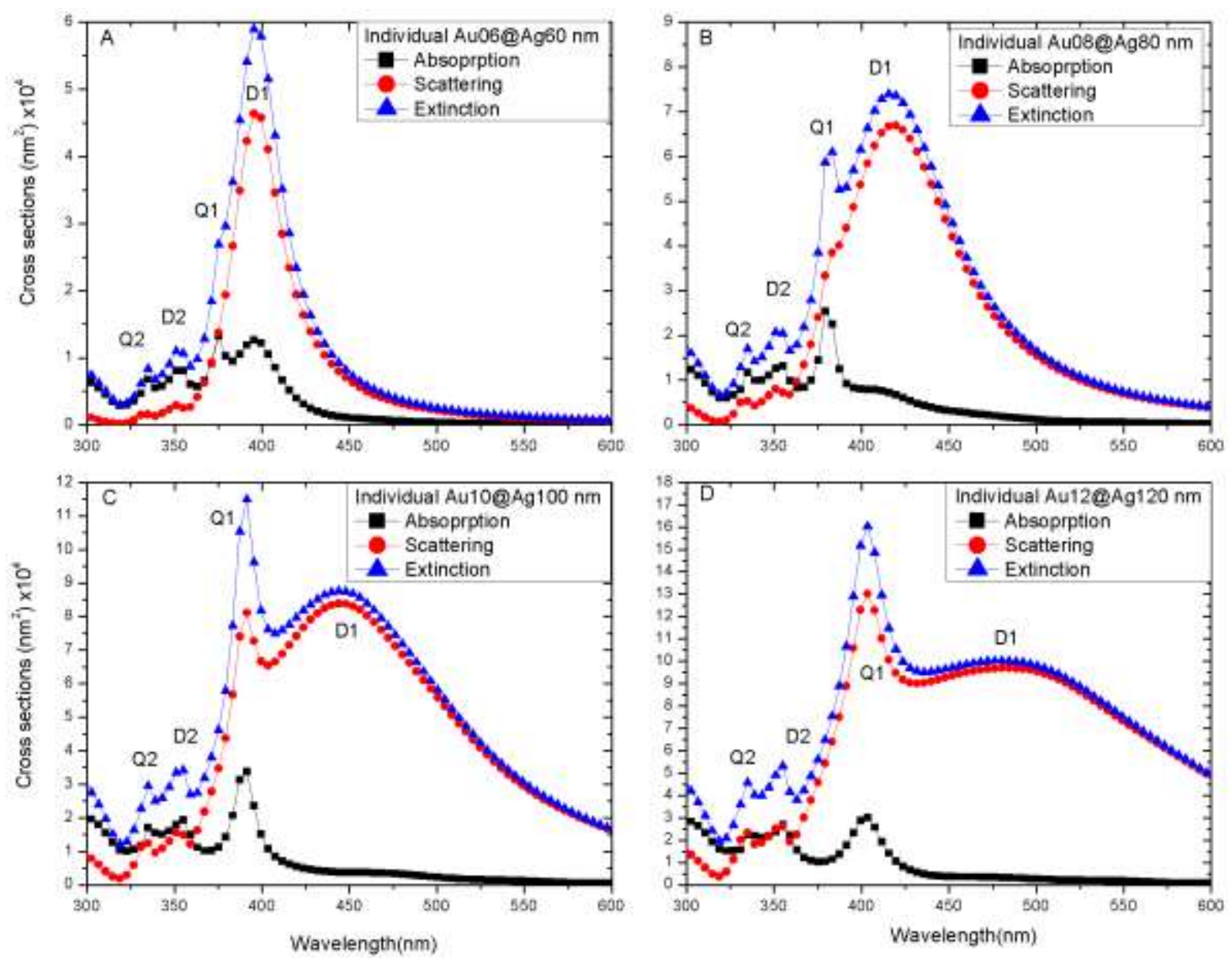

Fig. 1 (a) calculated cross sections of individual of core-shell Au@ Ag nanocube various shell sizes of (A) $60 \mathrm{~nm}$, (B) $80 \mathrm{~nm}$, (C) $100 \mathrm{~nm}$, (D) $120 \mathrm{~nm}$ at 10\% core/shell size ratio. The four main resonances dipolar D1, D2, and quadrupolar Q1, Q2 are assigned at the curves. 

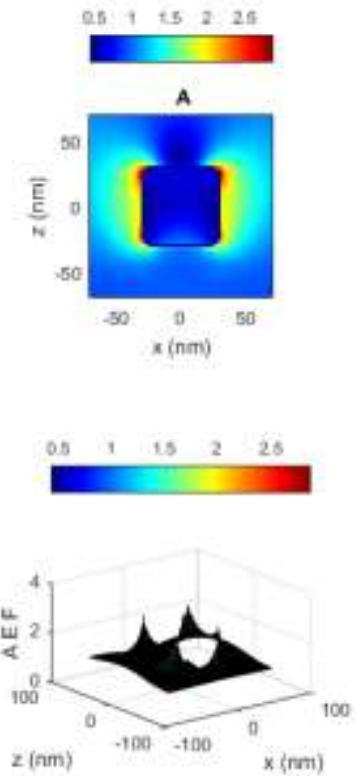
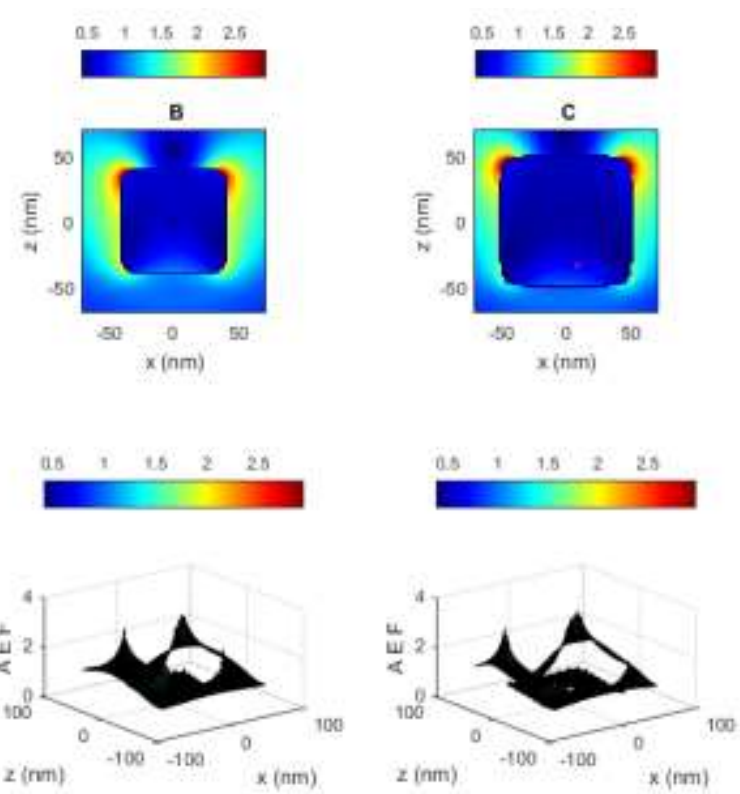

Fig. 1b Related field enhancement (upper panels) and the norm of electric field (A E F) (lower panels) of core-shell Au@ Ag nanocube various shell sizes of (A) $60 \mathrm{~nm}$, (B) $80 \mathrm{~nm}$, (C) $100 \mathrm{~nm}$, (D) $120 \mathrm{~nm}$ at $10 \%$ core/shell size ratio located in free space which is illuminated by a plane wave with $\mathrm{x}$-polarization obtained by the boundary element method. For the simulation of the induced electric field the resonance energy of $400 \mathrm{~nm}$ is used.
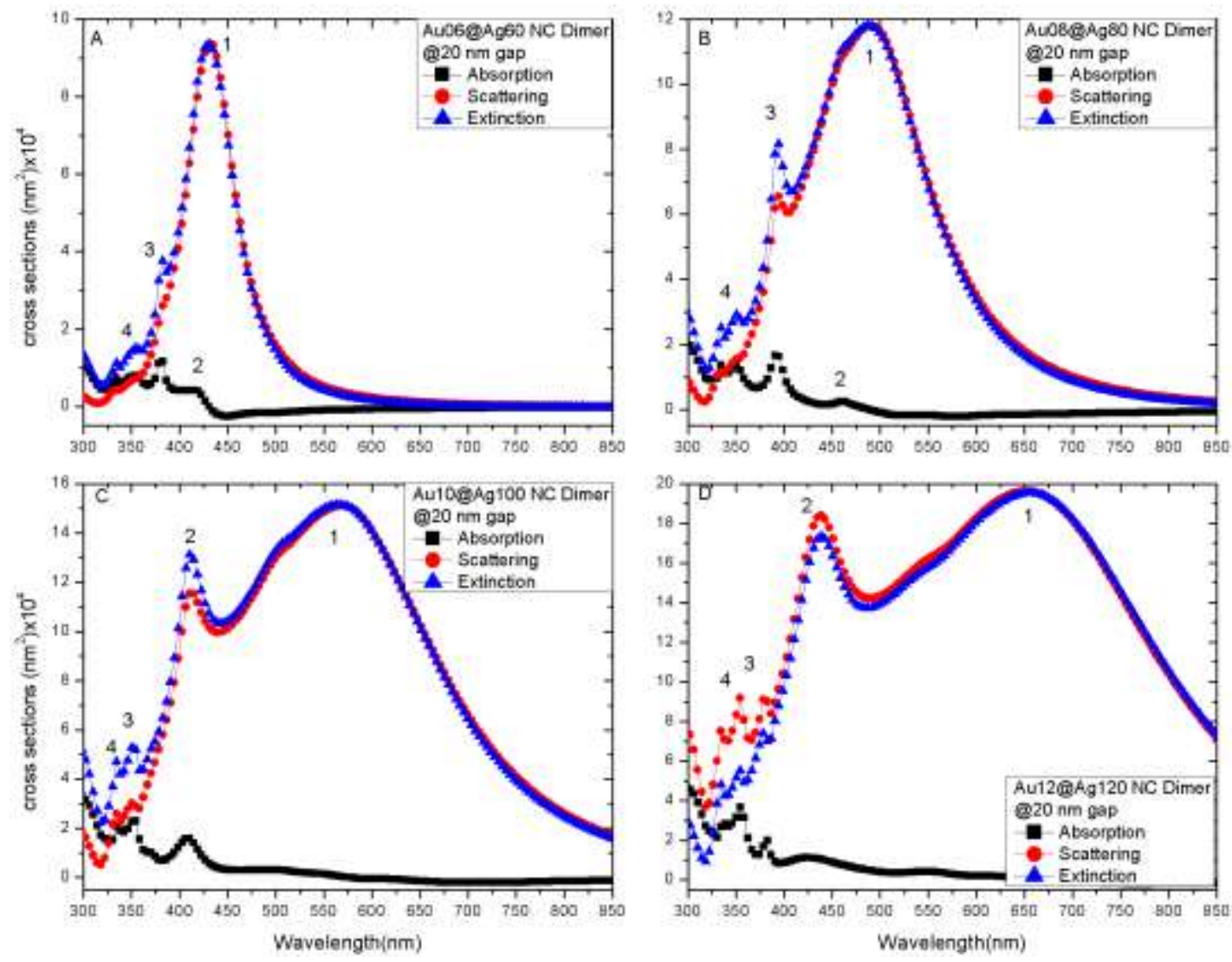
Fig. 2a calculated cross sections of large gap $(\mathrm{d}=20 \mathrm{~nm})$ core-shell Au@ Ag nanocube dimer of various shell sizes of (A) $60 \mathrm{~nm}$, (B) $80 \mathrm{~nm}$, (C) $100 \mathrm{~nm}$, and (D) $120 \mathrm{~nm}$ at $10 \%$ core/shell size ratio. The four main hybridized resonances modes, 1 (D1-D1), 2 (Q1-Q1), 3 (D2-D2), and 4 (Q2-Q2) are assigned at the curves.
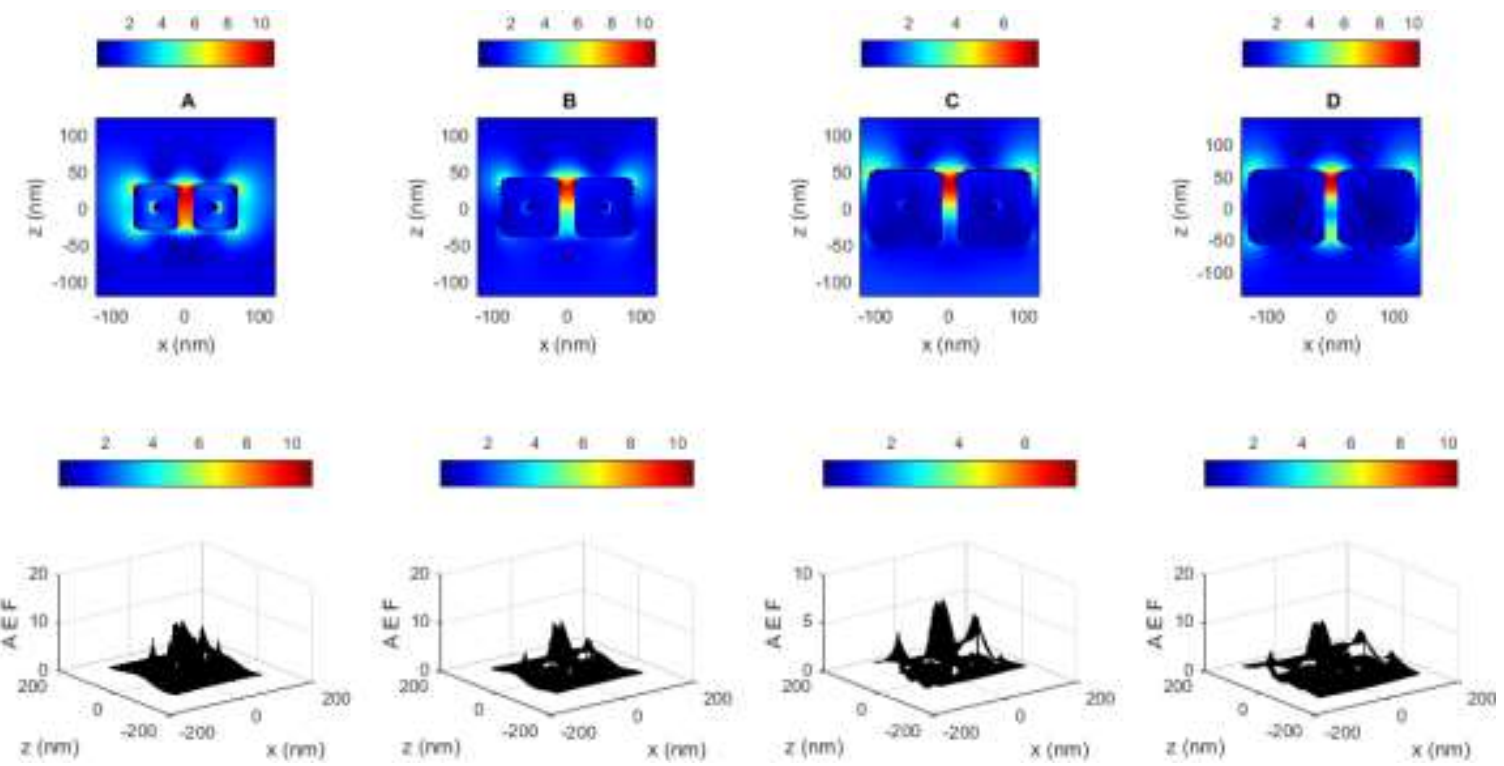

Fig. 2b Related field enhancement (upper panels) and the norm of electric field (A E F) (lower panels) of large gap $(\mathrm{d}=20 \mathrm{~nm})$ core-shell $\mathrm{Au} @ \mathrm{Ag}$ nanocube dimer of various shell sizes of (A) $60 \mathrm{~nm}$, (B) $80 \mathrm{~nm}$, (C) $100 \mathrm{~nm}$, and (D) $120 \mathrm{~nm}$ at 10\% core/shell size ratio located in free space which is illuminated by a plane wave with x-polarization obtained by the boundary element method. For the simulation of the induced electric field the resonance energy of $450 \mathrm{~nm}$ is used. 

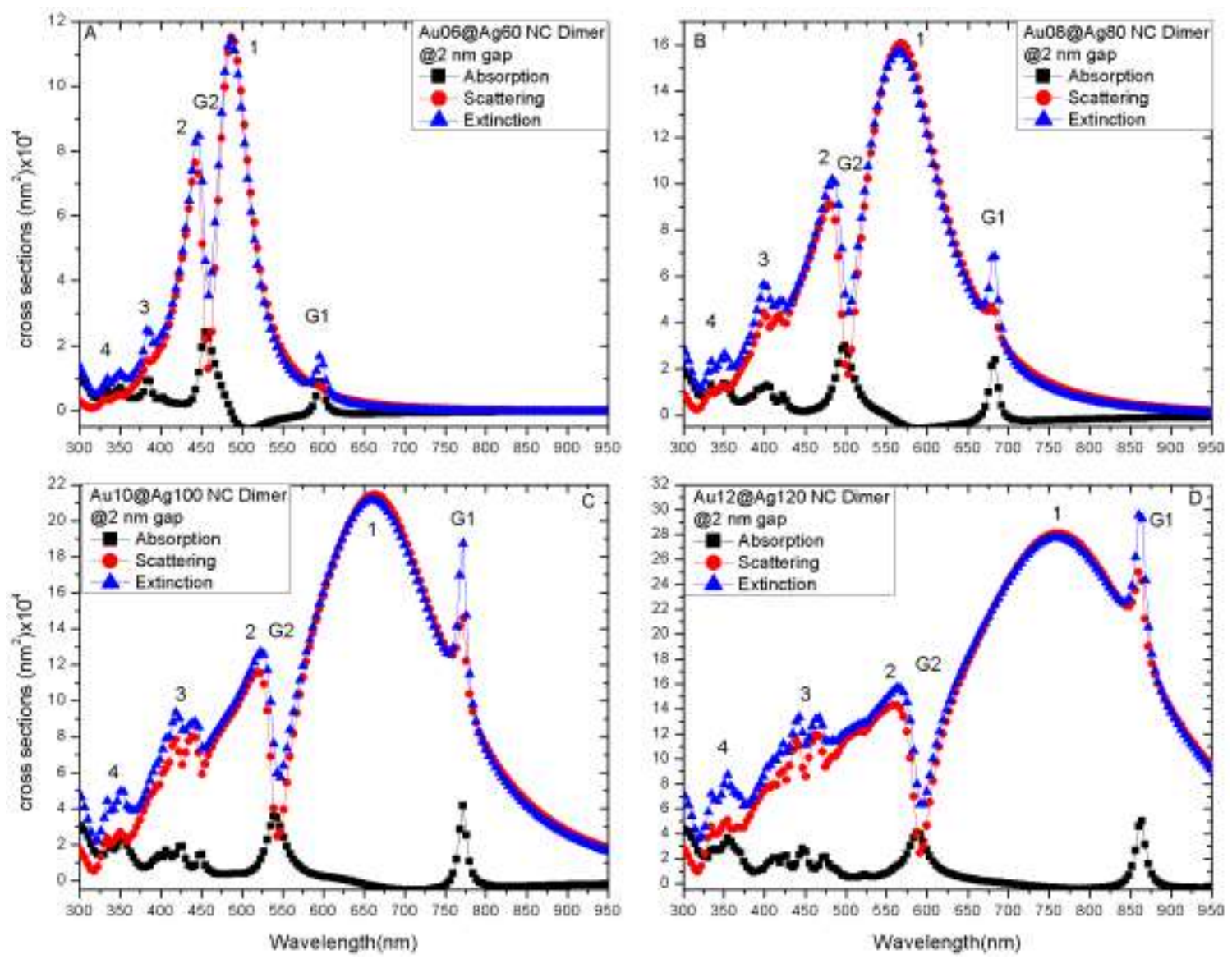

Fig. 3a calculated cross sections of small gap $(\mathrm{d}=2 \mathrm{~nm})$ core-shell $\mathrm{Au} @ \mathrm{Ag}$ nanocube dimer of various shell sizes of (A) $60 \mathrm{~nm}$, (B) $80 \mathrm{~nm}$, (C) $100 \mathrm{~nm}$, and (D) $120 \mathrm{~nm}$ at 10\% core/shell size ratio. The four main hybridized resonances modes, 1 (D1-D1), 2 (Q1-Q1), 3 (D2-D2), and 4 (Q2-Q2) and the two new resonances labeled as G1 and G2 are assigned at the curves.
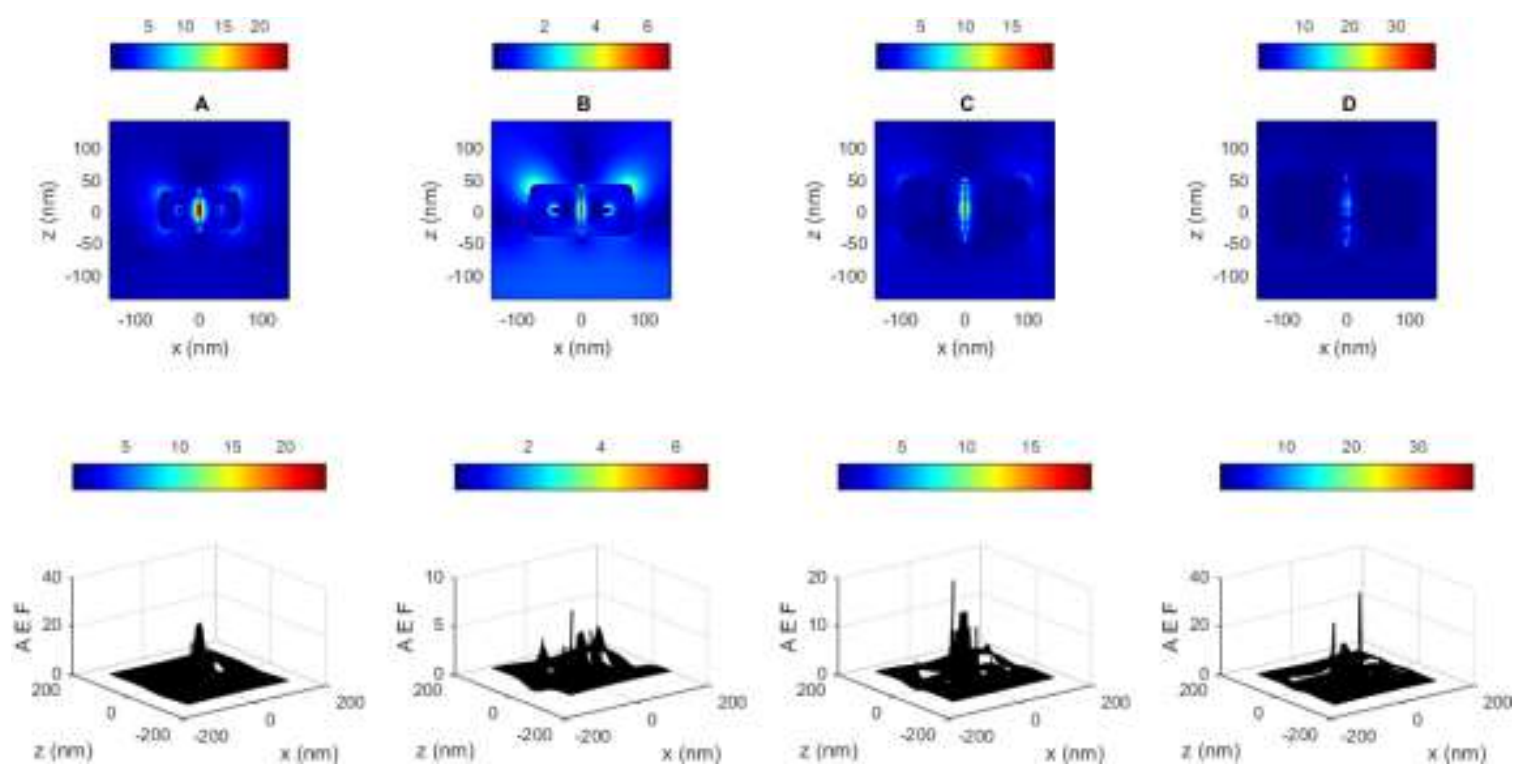
Fig. 3b Related field enhancement (upper panels) and the norm of electric field (A E F) (lower panels) of small gap ( $\mathrm{d}=2 \mathrm{~nm})$ core-shell Au@ Ag nanocube dimer of various shell sizes of (A) $60 \mathrm{~nm}$, (B) $80 \mathrm{~nm}$, (C) $100 \mathrm{~nm}$, and (D) $120 \mathrm{~nm}$ at 10\% core/shell size ratio located in free space which is illuminated by a plane wave with x-polarization obtained by the boundary element method. For the simulation of the induced electric field, the resonance energy of $500 \mathrm{~nm}$ is used.

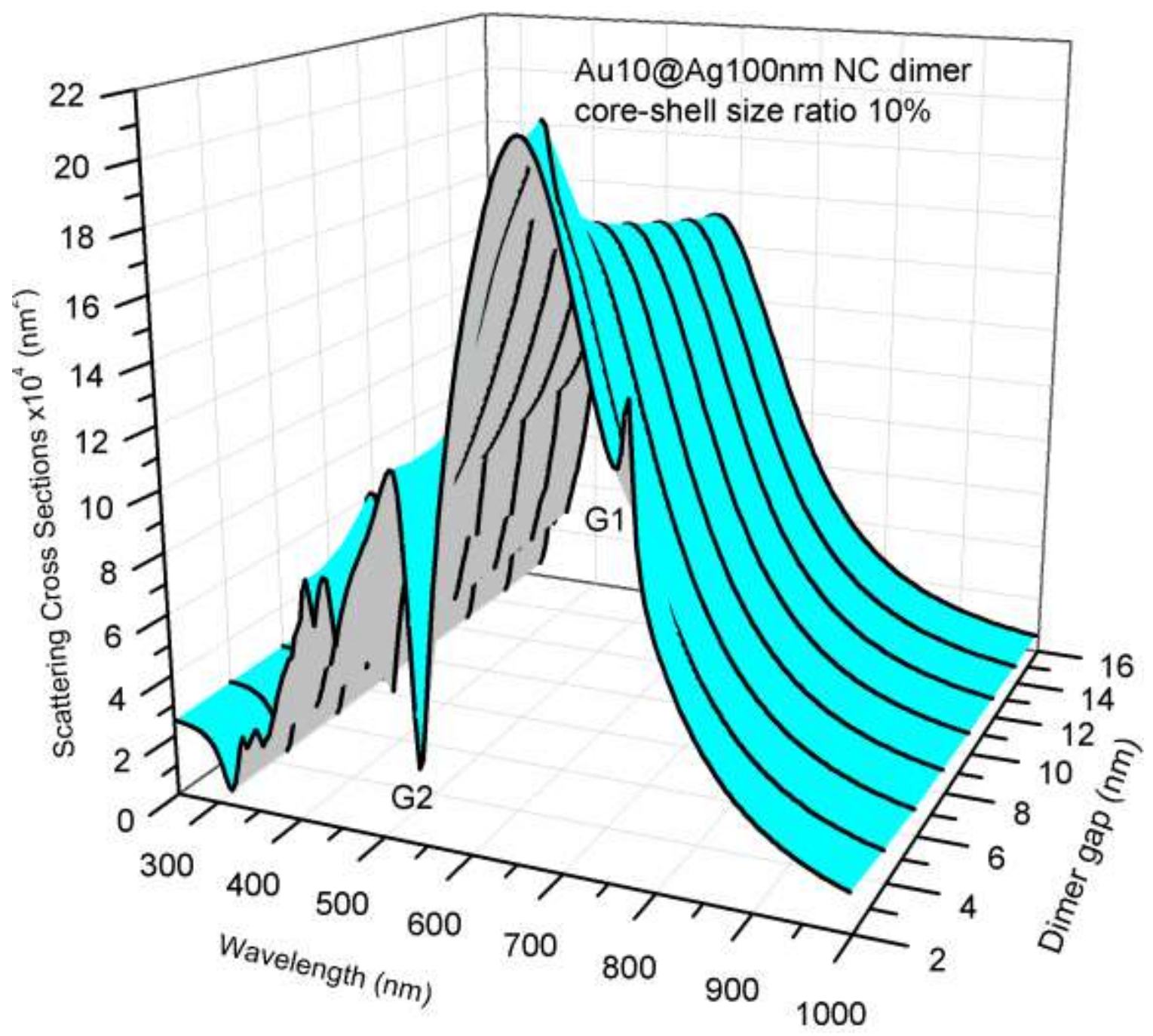

Fig. 4 Calculated scattering cross sections of Ag shell equal $100 \mathrm{~nm}$ at $10 \%$ core shell size ratio of gap separation vary from 2 to $16 \mathrm{~nm}$. The tracing of G2 interface dip with the variation of the gap separation is lost above gap separation equal $4 \mathrm{~nm}$. 

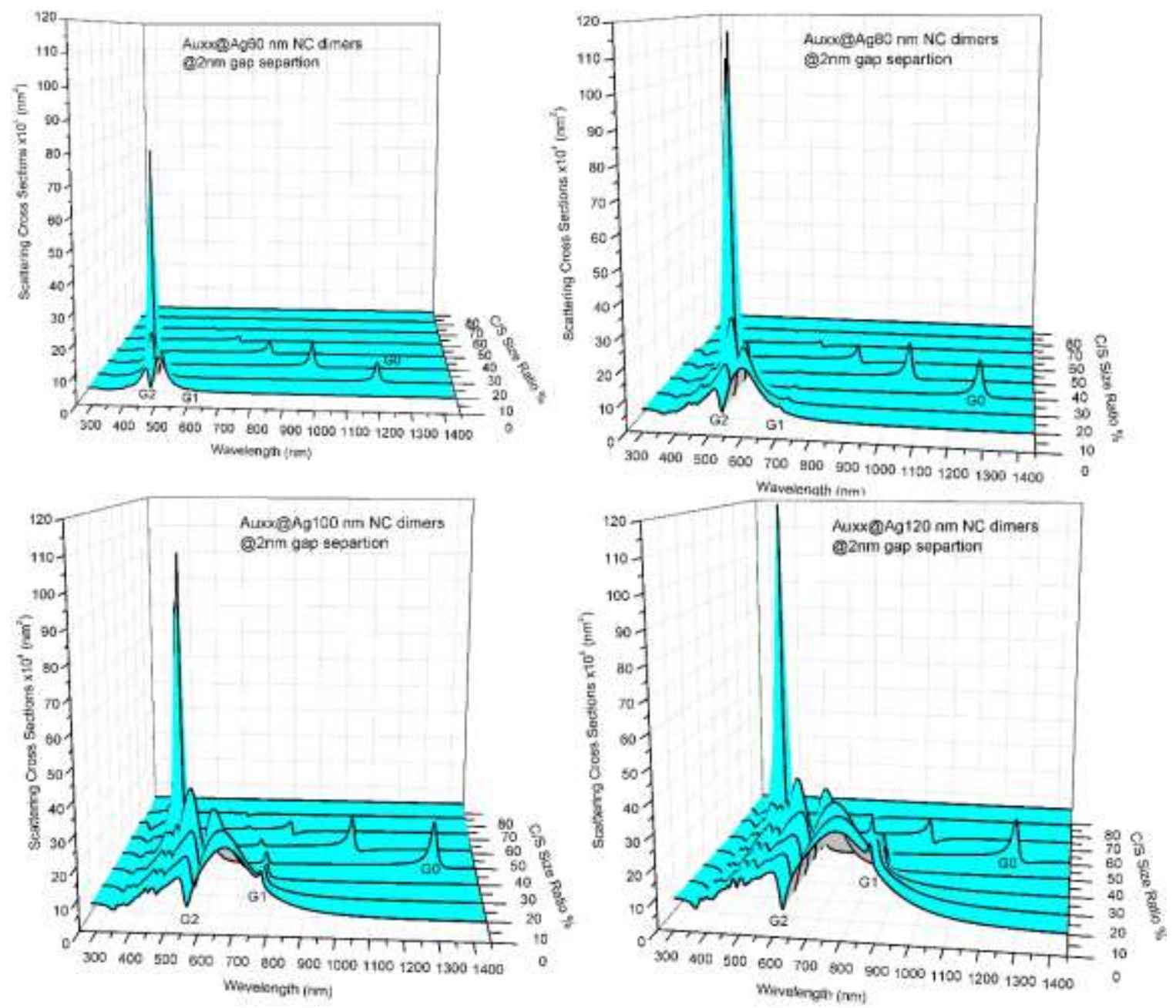

Fig. 5 Calculated scattering cross sections of Au@Ag nanocubes dimers of different shells sizes of $60 \mathrm{~nm}, 80 \mathrm{~nm}, 100 \mathrm{~nm}, 120 \mathrm{~nm}$ at different core/shell size ratio ranging from 10\% to $80 \%$ at small gap separation $(\mathrm{d}=2 \mathrm{~nm})$. The two resonances labeled as $\mathrm{G} 1$ and $\mathrm{G} 2$ and the new striking resonance peak labeled as G0 is assigned at the curves. Auxx represents the core size in $\mathrm{nm}$ see Table 1 for details. 

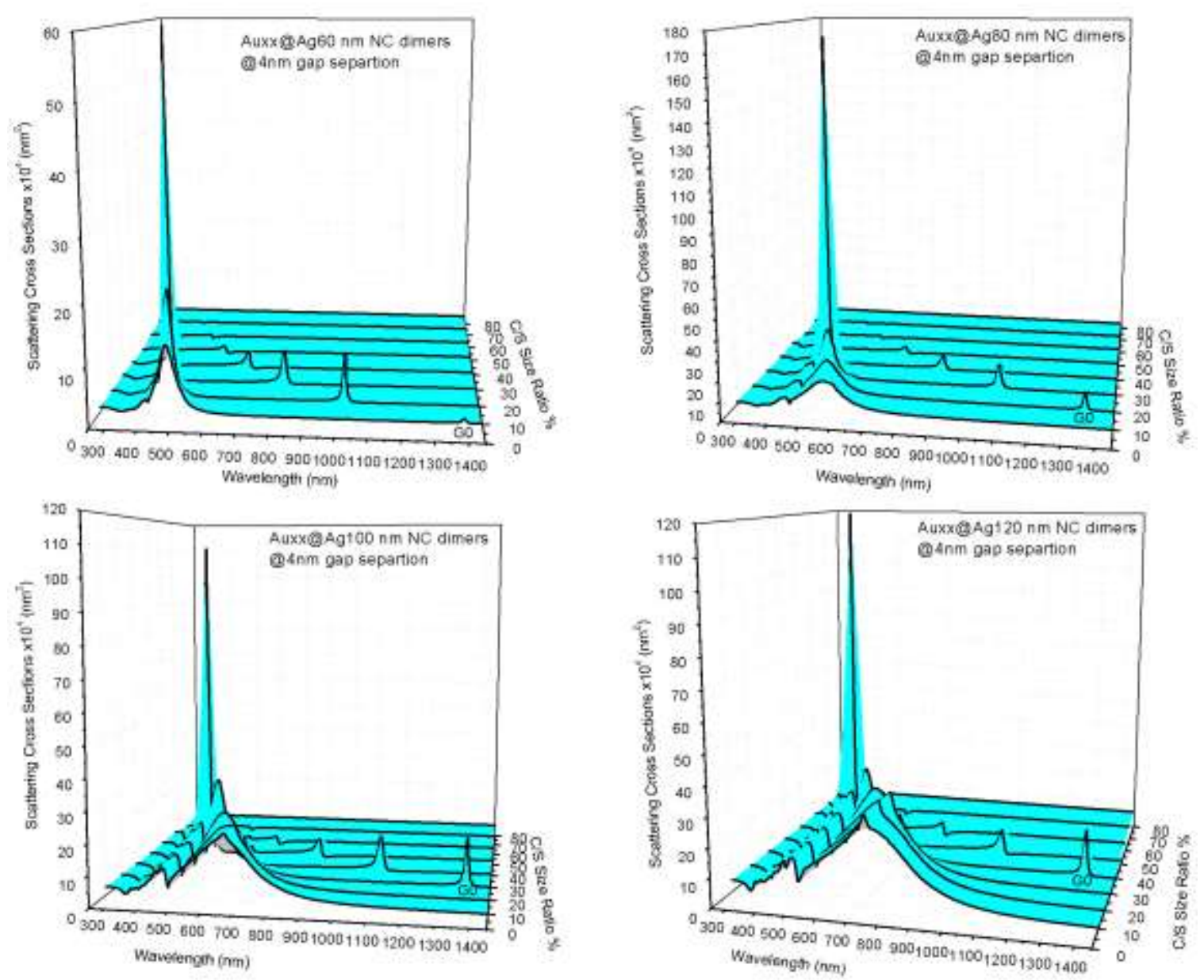

Fig. 6 Calculated scattering cross sections of Au@Ag nanocubes dimers of different shells sizes of $60 \mathrm{~nm}, 80 \mathrm{~nm}, 100 \mathrm{~nm}, 120 \mathrm{~nm}$ at different core/shell size ratio ranging from 10\% to $80 \%$ at gap separation $(\mathrm{d}=4 \mathrm{~nm})$. The new remarkable resonance peak labeled as $\mathrm{G} 0$ is assigned at the curves. Auxx represents the core size in nm see Table 1 for details. 

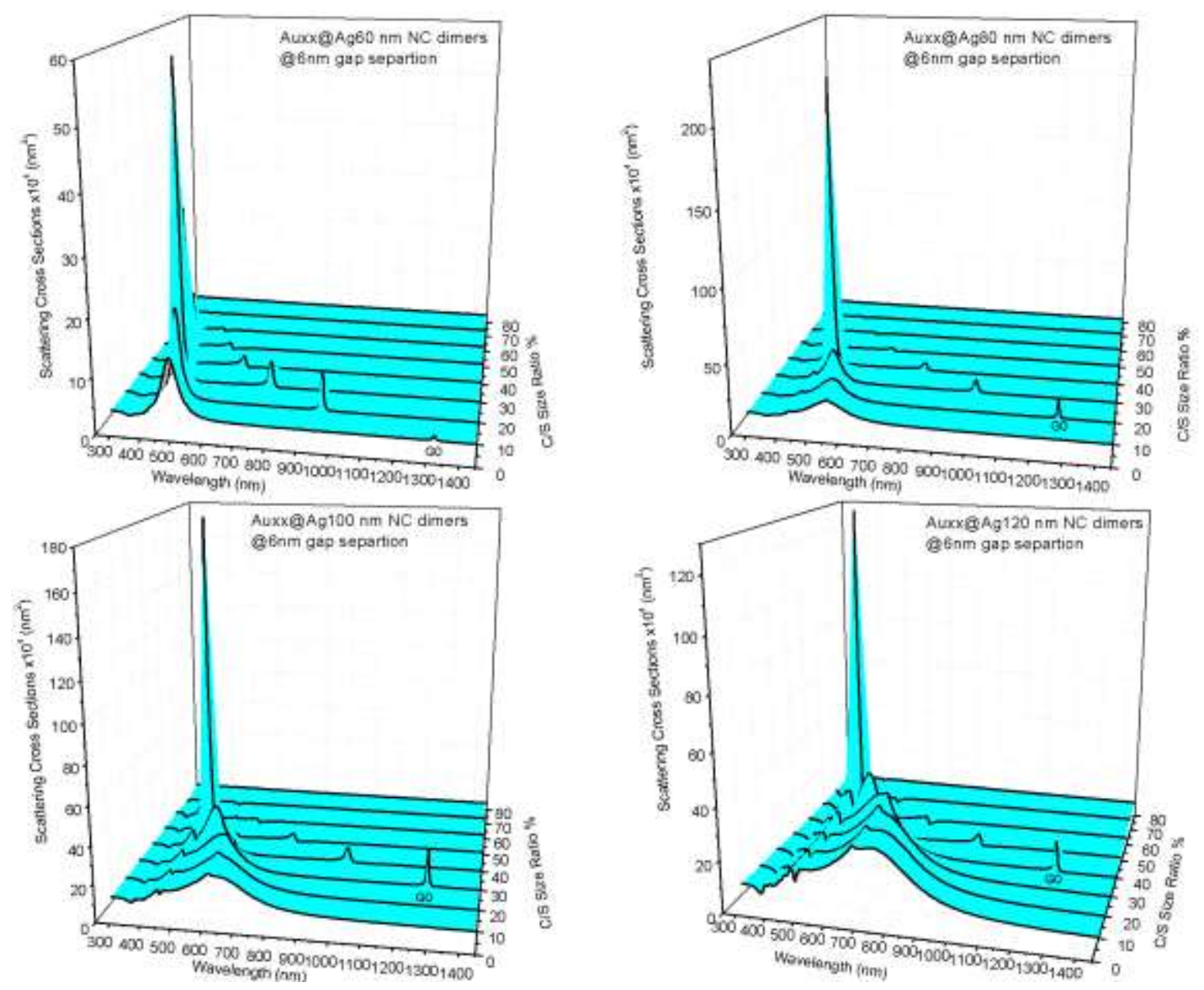

Fig. 7 Calculated scattering cross sections of Au@Ag nanocubes dimers of different shells sizes of $60 \mathrm{~nm}, 80 \mathrm{~nm}, 100 \mathrm{~nm}, 120 \mathrm{~nm}$ at different core/shell size ratio ranging from 10\% to $80 \%$ at gap separation $(\mathrm{d}=6 \mathrm{~nm})$. The new remarkable resonance peak labeled as $\mathrm{G} 0$ is assigned at the curves. Auxx represents the core size in nm see Table 1 for details. 


\section{Declaration}

\subsection{Funding}

This research was funded by Princess Nourah bint Abdulrahman University

Researchers Supporting Project number (PNURSP2022R38), Princess Nourah bint

Abdulrahman University, Riyadh, Saudi Arabia.

\subsection{Conflicts of interest/Competing interests}

The authors declare no conflicts of Interests

\subsection{Availability of data and material}

The data that support the findings of this study are available on request from the corresponding author

\subsection{Authors' contributions}

The manuscript was written through contributions of all authors.

\subsection{Ethics approval}

Not applicable

\subsection{Consent to participate}

Not applicable

\subsection{Consent for publication}

All authors read and approved the final manuscript for publication.

\section{References}

1. Manikandan, E., S.S. Princy, B.S. Sreeja, and S. Radha (2019) Structure Metallic Surface for Terahertz Plasmonics. Plasmonics 14 (6):1311-1319. doi:10.1007/s11468-019-00974-1

2. Ding, J., B. Arigong, H. Ren, J. Shao, M. Zhou, Y. Lin, and H. Zhang (2015) Dynamically Tunable Fano Metamaterials through the Coupling of Graphene Grating and Square Closed Ring Resonator. Plasmonics 10 (6):1833-1839. doi:10.1007/s11468-015-0003-6

3. Li, S., Y. Xie, J. Meng, W. Du, and J. Zhou (2020) Ultracompact and dynamically tunable plasmonic THz switch based on graphene microcavity metamaterial. Optical Review 27 (3):271-276. doi:10.1007/s10043-020-00589-6

4. Torabi, A. (2019) An efficient plasmonic waveguide mode solution using rational function fitting of Green's function. Optical Review 26 (2):278-282. doi:10.1007/s10043-01900498-3

5. Seifouri, M., M.A. Rouini, and S. Olyaee (2018) Design of a surface plasmon resonance biosensor based on photonic crystal fiber with elliptical holes. Optical Review 25 (5):555562. doi:10.1007/s10043-018-0447-y

6. Pellarin, M., J. Ramade, J.M. Rye, C. Bonnet, M. Broyer, M.-A. Lebeault, J. Lermé, S. Marguet, J.R.G. Navarro, and E. Cottancin (2016) Fano Transparency in Rounded Nanocube Dimers Induced by Gap Plasmon Coupling. ACS Nano 10 (12):11266-11279. doi:10.1021/acsnano.6b06406 
7. Zhou, H., D. Gao, and L. Gao (2018) Tunability of Multipolar Plasmon Resonances and Fano Resonances in Bimetallic Nanoshells. Plasmonics 13 (2):623-630. doi:10.1007/s11468017-0553-x

8. Bordley, J.A., N. Hooshmand, and M.A. El-Sayed (2015) The Coupling between Gold or Silver Nanocubes in Their Homo-Dimers: A New Coupling Mechanism at Short Separation Distances. Nano Letters 15 (5):3391-3397. doi:10.1021/acs.nanolett.5b00734

9. Yao, F., F. Li, Z. He, Y. Liu, L. Xu, and X. Han (2020) Tunable Fano Resonances in an UltraSmall Gap. Applied Sciences 10:2603

10. Cui, X., Y. Lai, F. Qin, L. Shao, J. Wang, and H.-Q. Lin (2020) Strengthening Fano resonance on gold nanoplates with gold nanospheres. Nanoscale 12 (3):1975-1984. doi:10.1039/C9NR09976J

11. Peña-Rodríguez, O., P. Díaz-Núñez, G. González-Rubio, V. Manzaneda-González, A. Rivera, J.M. Perlado, E. Junquera, and A. Guerrero-Martínez (2020) Au@Ag Core-Shell Nanorods Support Plasmonic Fano Resonances. Scientific Reports 10 (1):5921. doi:10.1038/s41598020-62852-9

12. Clark, B.D., C.R. Jacobson, M. Lou, D. Renard, G. Wu, L. Bursi, A.S. Ali, D.F. Swearer, A.-L. Tsai, P. Nordlander, and N.J. Halas (2019) Aluminum Nanocubes Have Sharp Corners. ACS Nano 13 (8):9682-9691. doi:10.1021/acsnano.9b05277

13. Li, G., H. Hu, and L. Wu (2019) Tailoring Fano lineshapes using plasmonic nanobars for highly sensitive sensing and directional emission. Physical Chemistry Chemical Physics 21 (1):252-259. doi:10.1039/C8CP05779F

14. Dhawan, A., S.J. Norton, M.D. Gerhold, and T. Vo-Dinh (2009) Comparison of FDTD numerical computations and analytical multipole expansion method for plasmonicsactive nanosphere dimers. Optics express 17 (12):9688-9703

15. Wei, G., Y. Shouwen, and H. Ganyun (2006) Finite element characterization of the sizedependent mechanical behaviour in nanosystems. Nanotechnology 17 (4):1118

16. Stannigel, K., M. König, J. Niegemann, and K. Busch (2009) Discontinuous Galerkin timedomain computations of metallic nanostructures. Optics Express 17 (17):14934-14947

17. Kane, Y. (1966) Numerical solution of initial boundary value problems involving maxwell's equations in isotropic media. IEEE Transactions on Antennas and Propagation 14 (3):302307. doi:10.1109/TAP.1966.1138693

18. Courant, R., K. Friedrichs, and H. Lewy (1967) On the Partial Difference Equations of Mathematical Physics. IBM Journal of Research and Development 11 (2):215-234. doi:10.1147/rd.112.0215

19. Ewe, W.-B., H.-S. Chu, and E.-P. Li (2007) Volume integral equation analysis of surface plasmon resonance of nanoparticles. Optics express 15 (26):18200-18208

20. Roopak, S., N. kumar Pathak, R. Sharma, A. Ji, H. Pathak, and R.P. Sharma (2016) Numerical Simulation of Extinction Spectra of Plasmonically Coupled Nanospheres Using Discrete Dipole Approximation: Influence of Compositional Asymmetry. Plasmonics 11 (6):1603-1612. doi:10.1007/s11468-016-0216-3

21. Kern, A.M. and O.J. Martin (2009) Surface integral formulation for 3D simulations of plasmonic and high permittivity nanostructures. JOSA A 26 (4):732-740

22. De Abajo, F.G. and A. Howie (2002) Retarded field calculation of electron energy loss in inhomogeneous dielectrics. Physical Review B 65 (11):115418

23. Liaw, J.-W. (2006) Simulation of surface plasmon resonance of metallic nanoparticles by the boundary-element method. Journal of the Optical Society of America A 23 (1):108116. doi:10.1364/JOSAA.23.000108 
24. Mäkitalo, J., S. Suuriniemi, and M. Kauranen (2011) Boundary element method for surface nonlinear optics of nanoparticles. Optics Express 19 (23):23386-23399. doi:10.1364/OE.19.023386

25. Becker, J., A. Trügler, A. Jakab, U. Hohenester, and C. Sönnichsen (2010) The Optimal Aspect Ratio of Gold Nanorods for Plasmonic Bio-sensing. Plasmonics 5 (2):161-167. doi:10.1007/s11468-010-9130-2

26. Hohenester, U. and A. Trügler (2012) MNPBEM - A Matlab toolbox for the simulation of plasmonic nanoparticles. Computer Physics Communications 183 (2):370-381. doi:10.1016/j.cpc.2011.09.009

27. Johnson, P.B. and R.W. Christy (1972) Optical Constants of the Noble Metals. Physical Review B 6 (12):4370-4379. doi:10.1103/PhysRevB.6.4370

28. Ahmad, M. (2018) Simulations of the Surface Plasmon Resonances of Au@Ag Core-Shell Nanocubes: Effect of Core-Shell Size Ratio. Plasmonics 13 (3):981-990. doi:10.1007/s11468-017-0596-z

29. Zhang, S., K. Bao, N.J. Halas, H. Xu, and P. Nordlander (2011) Substrate-Induced Fano Resonances of a Plasmonic Nanocube: A Route to Increased-Sensitivity Localized Surface Plasmon Resonance Sensors Revealed. Nano Letters 11 (4):1657-1663. doi:10.1021/nl200135r

30. Walther, R., S. Fritz, E. Müller, R. Schneider, D. Gerthsen, W. Sigle, T. Maniv, H. Cohen, C. Matyssek, and K. Busch (2016) Coupling of Surface-Plasmon-Polariton-Hybridized Cavity Modes between Submicron Slits in a Thin Gold Film. ACS Photonics 3 (5):836-843. doi:10.1021/acsphotonics.6b00045 